\title{
Potencialización del ecoturismo inteligente en la finca de conservación "Rivera de los vencejos", cantón Piñas, provincia de El Oro-Ecuador
}

\section{Potentialization of intelligent ecotourism in the conservation farm "Rivera de los vencejos", canton Piñas, province of El Oro- Ecuador}

\author{
Lic. Jessica Ivonne Lalangui Ramírez. Mgs. ${ }^{1}$ \\ jlalangui@umet.edu.ec
}

Recibido: 1/12/ 2016, Aceptado: 1/02/2017

\begin{abstract}
RESUMEN
La Finca de Conservación "Rivera de los Vencejos", constituye un lugar de conocimiento natural y de conservación del ecosistema, reflejando en su esplendor diferentes especies de flora y fauna que forman parte de su entorno paisajístico. Resaltan sus recursos naturales con magníficos paisajes y hermosas cascadas. No obstante, se detectó una deficiente comunicación en márquetin, que resalte sus atractivos para la captación de turismo, por lo que esta investigación se enfoca en potencializar una oferta que permita la diversificación de actividades turísticas, mejorando la competitividad, conservando los recursos naturales y respetando la autenticidad sociocultural de las comunidades anfitrionas y del ecosistema de la zona; basado en un turismo inteligente que contribuya al desarrollo local involucrando a la comunidad e incentivando además, la visita del turista nacional e internacional. Para ello, se aplicaron métodos teóricos y empíricos de observación y entrevista, investigación de campo que diagnosticó recursos del área, así como consulta de materiales legislativos, todo lo cual permitió arribar a propuestas de solución y elaboración de un plan de márquetin, resultados que fueron acogidos con entusiasmo por la directiva.
\end{abstract}

Palabras clave: finca de conservación, ecoturismo inteligente, desarrollo local, recursos naturales, turismo sostenible

\section{ABSTRACT}

The "Rivera de los Vencejos" Conservation Estate is a place of natural knowledge and conservation of the ecosystem, reflecting in its splendor different species of flora and fauna that are part of its landscape environment. They highlight their natural resources with magnificent landscapes and beautiful waterfalls. However, there was a lack of communication in marketing, highlighting its attractiveness to attract tourism, so this research focuses on potentiating an offer that allows the diversification of tourist activities, improving competitiveness, conserving natural

\footnotetext{
1 Docente de la carrera de Turismo de la Universidad Metropolitana (UMET), extensión Machala. Ecuador.
} 
activities, improving competitiveness, conserving natural resources and respecting the socio-cultural authenticity of the host communities and the ecosystem of the area; Based on intelligent tourism that contributes to local development involving the community and also encourages the visit of national and international tourists. For this, theoretical and empirical methods of observation and interview were applied, field research that diagnosed resources of the area, as well as consultation of legislative materials, all of which allowed to arrive at proposals of solution and elaboration of a plan of marketing, results that were Welcomed with enthusiasm by the directive.

Keywords: conservation farm, intelligent ecotourism, local development, natural resources, sustainable tourism

\section{Introducción}

Ante el desarrollo turístico mundial, Ecuador cuenta con una variedad muy extensa que podría significar grandes recursos económicos.

En este sentido en la provincia de El Oro existe una gran diversidad de clima, fauna que permite potencializar el turismo. Por esta razón resulta conveniente promocionar turísticamente cada uno de ellos.

Gracias a su ubicación geográfica se caracteriza por su clima templado, que brinda un ambiente de costa y a su vez de sierra, muy preciado por sus habitantes y turistas nacionales e internacionales.

En la Finca de Conservación "Rivera de los Vencejos", se concentra gran variedad de ecosistemas con peculiares atractivos naturales, que resulta un indudable potencial turístico, aún sin explotar sosteniblemente con el entorno. Es menester lograr que los pobladores de las comunidades anfitrionas de la zona, contribuyan a la conservación de la finca y su entorno, de tal forma que no exista impacto hacia el medio ambiente, proponiendo planes de mejora, sociales y culturales. El presente proyecto de estudio de campo e investigación, realizó actividades para la potencialización de un Ecoturismo Inteligente en dicha zona.

Es importante entonces realizar una campaña intensiva por diferentes medios gubernamentales, locales o de cualquier otra índole, para promover la actividad turística del cantón Piñas, exactamente en la Finca antes mencionada.

El ministerio de turismo está colaborando mediante conferencias sobre el ecoturismo y el turismo sostenible, para de esa forma brindar un espacio de aprendizaje, debate, interacción, con expertos internacionales y la industria turística en Ecoturismo y Turismo Sostenible, para de esa forma contribuir al posicionamiento de Ecuador como un lugar biodiverso comprometido con la conservación del medio ambiente y una oferta turística de calidad.

Dicha finca, como muchos sitios exuberantes del Ecuador, en los que falta aún mucho por hacer en materia de lanzamiento al turismo sostenible, no cuenta con un programa propagandístico de comunicación consolidado, que propicie la avenida de turistas, tanto locales, nacionales y preferentemente internacionales.

El principal problema a resolver se enfoca en ¿Cómo contribuir al alza del Turismo en la Finca de Conservación "Rivera de los Vencejos" ?, para ello el objetivo se centra 


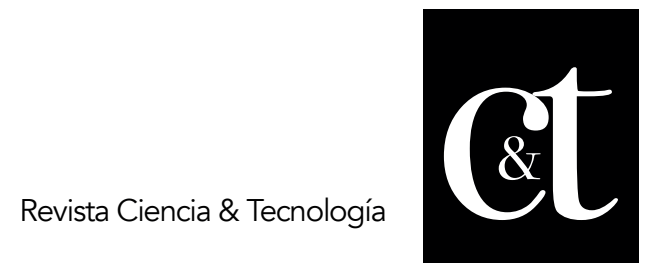

No. 14, 30 de abril de 2017

ISSN impreso: 1390 - 6321

en: Potencializar una oferta que permita la diversificación de actividades turísticas, conservando los recursos naturales y respetando la autenticidad sociocultural de las comunidades anfitrionas y del ecosistema de la zona, mediante un plan de márquetin basado en un turismo inteligente.

\section{Materiales y métodos}

Breve caracterización del área de estudio Hacienda "Rivera De Los Vencejos". EL sitio se creó entre 2008 y 2009 por iniciativa de un grupo de entusiastas del turismo y el apoyo de diferentes personas que fueron aportando con sus ideas, así como, a las bondades del lugar, ya sea por sus tres cascadas, de varios metros de altura y profundidad o por tener en una de las cascadas el hábitat para anidación de los Apus apus sp. (Vencejos). Convirtiéndose en un paraíso ornitológico, atrayendo a turistas nacionales y extranjeros. Dentro de la Hacienda "Rivera de los Vencejos" existe un mirador donde se puede realizar camping, una cabaña rústica que cuenta con servicios básicos con un número de 4 habitaciones, un comedor, un baño y áreas verdes para el confort de los visitantes.

Ubicación y límites: El sitio Ribera de Los Vencejos, se encuentra ubicado en la provincia de El Oro, el cantón Piñas en el sitio La Florida de la parroquia la Bocana. La finca tiene una superficie total de 15 hectáreas en las que se encuentran varios atractivos como cascadas, bosques, el hábitat natural de los vencejos, entre otros. ¿Cómo llegar?: Desde la parroquia Saracay, se toma la ruta Saracay-Piedras-El Carmen, se recorren aproximadamente $5 \mathrm{~km}$., luego desde el Sitio El Carmen se continúa por la ruta. El Carmen-Cañas, pasando el puente toma una vía a mano izquierda y recorre aproximadamente $2 \mathrm{~km}$. hasta llegar a la finca.

Técnicas: En esta investigación se emplearon las siguientes técnicas:

- Investigación de Campo: se planificó con el Sr. Modesto Rivera propietario de la Finca de Conservación "Rivera de los Vencejos" ubicada en el sitio La Florida, donde se recopiló toda la información para llevar a cabo el proyecto. Además, se realizó una breve entrevista para obtener información concisa.

- Documentos de información: se utilizó una guía de observación que permitió identificar los atractivos paisajísticos.

- Fuentes secundarias: libros, revistas, artículos científicos, internet y proyectos anteriores.

\section{Ecoturismo}

El ecoturismo, según algunos analistas, ha sido relacionado frecuentemente con el turismo de aventura, el cual, en la mayoría de los casos ha sido utilizado para designar actividades de índole ecológica, que en ocasiones han permitido llamar la atención de las personas que aspiran a practicar una verdadera actividad ecoturística. Esta situación hace del ecoturismo una forma más del turismo masivo corriente, desvirtuando su esencia, así como los objetivos y beneficios que ofrece a las comunidades, in situ, o aledañas a las áreas protegidas y a los turistas, al igual que a la conservación de los recursos naturales y a la protección ambiental (Bulla, 2013).

Por esta razón a manera de ilustración, se presentan los aspectos que el ecólogo norteamericano George Wallace (1992), consideró como fundamentales para identificar y rubricar lo que es, en sí, el ecoturismo:

1. Lograr que las áreas naturales sean percibidas tanto como "un hogar para todos nosotros", en un sentido planetario y "un hogar para los residentes locales en su significado especifico". 


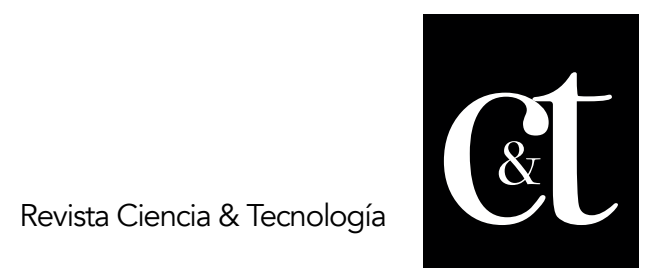

No. 14, 30 de abril de 2017

ISSN impreso: 1390 - 6321

2. Permitir minimizar los impactos negativos, tanto en el medio ambiente natural, como en los habitantes locales.

3. Contribuir a la gestión de las áreas protegidas y a mejorar los vínculos entre las comunidades locales y los administradores de dichas áreas protegidas.

4. Propiciar beneficios económicos y de otra índole para los habitantes del lugar y maximizar su participación en el proceso decisorio que determina del tipo y la cantidad de turismo que debe ocurrir.

5. Promover una autentica interacción entre visitantes y los anfitriones, así como un interés genuino en el desarrollo sostenible y la protección de áreas naturales, tanto en el país que se visita, como en el país de origen del turista.

6. Suplementar o completar prácticas tradicionales como agricultura, ganadería, pesca, sistemas sociales otras (alternativas de trabajo y complemento de dichas actividades), sin marginarlas o intentarlas reemplazar, fortaleciendo de esta manera la economía local al hacerla menos susceptible a cambios bruscos internos y externos. 7. Ofrecer oportunidades especiales para que los habitantes locales y los empleados de agencias turísticas, también utilicen de manera sostenibles las áreas naturales y aprender más sobre los atractivos ecoturísticos que se ofrece al visitante.

Coincidiendo con lo anterior, Wearing \& Neil (2000) encuentran que constituyen aspectos importantes a considerar para un turismo sostenible de naturaleza y desarrollo local, que resalte atractivos, actividades e idiosincrasia comunitaria.

\section{Turismo comunitario en Ecuador}

En la década de los 80 , nace el Turismo Comunitario como una respuesta que permite a las comunidades generar una alternativa para mejorar los ingresos familiares, recuperar y valorizar su cultura e identidad y el manejo sostenible de los recursos naturales. Producto de la creciente orientación de los turistas hacia áreas naturales en búsqueda de nuevos estímulos/ retos, en su deseo de estar en plena naturaleza, pues es cada día más difícil encontrar esta sensación en sus países de origen, así como en una sensibilidad cada vez mayor acerca de la situación y los problemas del medio ambiente. Como una alternativa de obtener nuevas formas de ingresos y reducir los niveles de pobreza diversas comunidades se integraron para generar emprendimiento de Turismo Comunitario en la localidad, para con ello: ejercer una adecuada defensa y manejo de sus tierras y territorios, la revalorización cultural, fortalecimiento organizativo, distribución equitativa de los beneficios generados y seguridad alimentaria (MINTUR, 2017).

FODA del turismo comunitario en el Ecuador EL diagnóstico de Turismo Comunitario en el Ecuador fue formulado por el Ministerio de Turismo, para lo cual dentro del PLANDETUR 2020 (MINTUR, 2017) se considera las siguientes fortalezas, oportunidades, debilidades y amenazas:

Fortalezas;

- Fuerte componente natural y cultural en los territorios

- Gestión local participativa

Oportunidades:

- Demanda de productos diversificados

- Reduce índices de migración

- Nuevos nichos de mercado

Debilidades: 


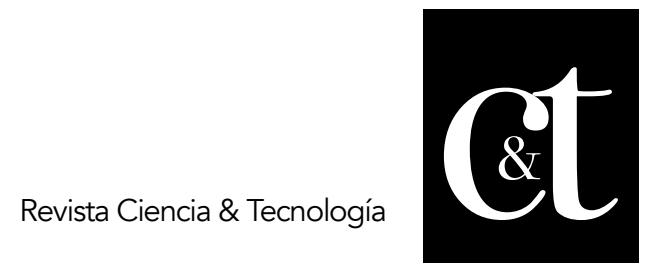

No. 14, 30 de abril de 2017

ISSN impreso: 1390 - 6321

- Falta de canales de comercialización

- Oferta carente de estándares de calidad básicos

- Escasa gestión administrativa financiera

Amenazas:

- Falta de regularización legal

- Empresa privada que comercializa operaciones comunitarias.

Como resaltan estos estudios del MINTUR, desde inicios de la década pasada, ya se conocen, en lo fundamental, las principales debilidades del turismo comunitario ecuatoriano, para lo cual se han venido trazando estrategias.

\section{Turismo Inteligente}

Un destino turístico innovador, consolidado sobre una infraestructura tecnológica de vanguardia, que garantiza el desarrollo sostenible del territorio turístico, accesible para todos, que facilita la interacción e integración del visitante con el entorno e incrementa la calidad de su experiencia en el destino, a la vez que mejora la calidad de vida del residente. La consecuencia de la reconversión es la estimulación y el incremento de la competitividad a través de la capacidad innovadora, que repercute en una mejora de la percepción del destino, generando márgenes superiores de la calidad de vida de los residentes (SEGITTUR, 2012).

La intención de convertirse en un DTI supone el establecimiento de una estrategia de revalorización del destino que permita aumentar su competitividad, mediante un mejor aprovechamiento de sus atractivos naturales y culturales, la creación de otros recursos innovadores, la mejora en la eficiencia de los procesos de producción y distribución que finalmente impulse el desarrollo sostenible y facilite la interacción del visitante con el destino (SEGITTUR, 2012).

¿Qué son los vencejos?

Su nombre científico: Apus apus sp., es un ave diseñada para volar desde el primer instante que se lanzan al aire, con alas aeronautas, cola corta horquillada, boca desproporcionada, pero de pico pequeño que le permite capturar insectos voladores, sus patas poco funcionales, ya que han sufrido una involución debido a su adaptación netamente al aire. No posee pulgar, pero si unas garras muy fuertes con las cuales cuelgan de paredes rocosas, su plumaje negro con pequeñas manchas blancas. Sus alas pueden llegar a medir de 42 a 48 centímetros que le dan elegancia y una apariencia a media luna al momento de volar. Su vida sexual reproductiva e incluso alimentación lo hacen en el aire, aterrizado solo una vez al año las hembras para anidar los polluelos, los que después de pocos días emprenden el vuelo sin volver a tierra hasta alcanzar la madurez sexual en el caso de las hembras.

\section{Resultados y discusión}

Resumen de resultados de observaciones, entrevista y diagnóstico de campo. Los resultados que se han obtenido después de haber realizado la observación del lugar, la entrevista y la ficha de observación; se puede detallar que esta es una zona muy acogedora para los turistas que tienen un alma aventura y buscan experiencias nuevas, hay una gran cantidad de espacios verdes, avistamiento de aves, zonas de descanso, zonas de disfrute, entre otros atractivos.

Considerando que esta zona recibe pocos turistas en la actualidad, debido a que no existe una página con información precisa y detallada del lugar, o una Aplicación para dispositivos tecnológicos (APP), ofreciendo los diferentes servicios del lugar, y con 
aquellos fondos que genere la misma, implementar lo que sería el acondicionamiento de cabañas rusticas; para potencializar este lugar, se necesitó de varios recursos como:

- Publicidad en línea

- Señaléticas de tránsito

- Creación de APP del lugar a potenciar

- Inclusión del lugar en la Página web del Ministerio de Turismo

Propuesta de potencialización para oferta basado en turismo inteligente. -

- Creación de recursos tecnológicos, apoyándose en la Universidad Metropolitana (UMET), sede Machala, dentro de sus proyectos de vinculación con la sociedad, como son: Publicidad en línea, Página web del lugar a potenciar.

- Solicitar al Gobierno Autónomo Descentralizado Municipal el arreglo de la vía principal para la instalación de señalética de tránsito y previamente solicitar al Ministerio de Turismo la señalética turística para la hacienda.

- Creación de un APP, que permita promover al lugar, con apoyo también de la UMET. - Solicitar al Ministerio de Turismo, programa de capacitación con profesionales para brindar asesorías sobre emprendimiento, manipulación de alimentos, turismo comunitario y atención al cliente, entre otros temas, tanto a los trabajadores del área como a los habitantes del lugar, para que la zona pueda tener un crecimiento económico.

Se propone, además:

1. Realizar un estudio sobre la capacidad de personas que pueden ingresar en la zona, para que de esa forma no exista un impacto al lugar.

2. Aumentar el personal para el cuidado y limpieza de la zona.

3. Realizar un seguimiento de las actividades, tanto turísticas como comerciales en el Sitio La Florida, pueblo donde se realizan actividades comerciales antes de llegar a la Hacienda "Rivera de los Vencejos", para de esa forma, verificar si hay un incremento de los beneficios económicos y sociales.

\section{Conclusiones}

Las principales causas que inciden según la investigación en la escasa afluencia turística, son la falta del arreglo de la vía principal para la instalación de señalética de tránsito, la falta de la señalética turística hacia la hacienda, y la insuficiente información acerca del lugar turístico. Para materializar esta propuesta, el dueño de la hacienda debe realizar una solicitud hacia el Ministerio de Turismo para constar entre los lugares que se encuentran publicitados en su página web. Finalmente, captar la atención de operadores turísticos grandes que incluso podrían iniciar el flujo de turistas extranjeros al Sitio.

\section{Referencias bibliográficas}

Bulla, L. H. (2013). Ecoturismo: oferta y desarrollo sistemático regional. Bogota: Ecoe Ediciones.

Criollo, C. (s.f.). Blog ESPOL. Recuperado el 15 de 12 de 2016, de http://blog.espol.edu.ec/giscacri/historia/

GAD Municipal Piñas. (2016). Recuperado el 15 de 12 de 2016, de http://pinas.gob.ec/turismo/item/356-finca-de-conservacion-rivera-de-los- 


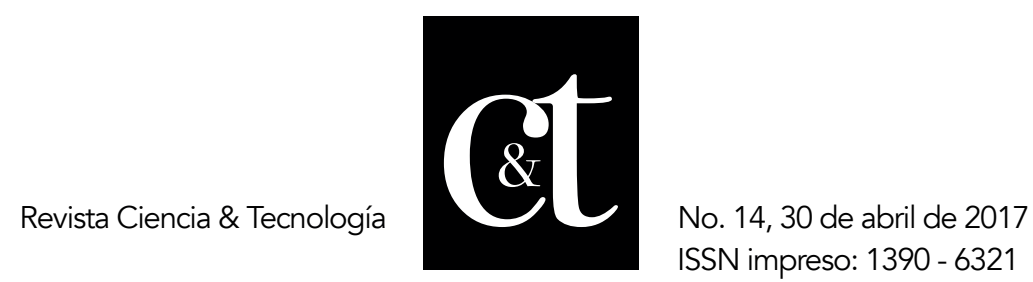

vencejos.html

Galeon (2014). Galeon.com. Recuperado el 15 de12 de 2016, http://turismooro.galeon.com/

Ministerio de Turismo MINTUR (2007). PLANDETUR 2020. Quito. Disponible https://www.ecured.cu/Provincia_de_El_Or

Sociedad Mercantil Estatal para la Gestión de la Innovación y las Tecnologías Turísticas SEGITTUR (2012). Destinos Turísticos Inteligentes. Recuperado el 15 de 12 de 2016, de_http://www.segittur.es/ es/proyectos/proyectodetalle/Destinos-Tursticos-Inteligentes- 00006/\#.WJSzTdLhDIU

Wallace, G. (1992). Real Ecotourism: Assiting protected areas managers and getting benefits to local people. USA: Colorado State University.

Wearing, S. \& Neil, J. (2000). Ecoturismo: impacto, tendencias y posibilidades. Madrid: Síntesis. 